\title{
Behavioral Issues in Asthma Management
}

\author{
Randall Brown, MD, MPH* \\ Summary. Despite advances in drug treatment, outcomes in asthma remain unsatisfactory. \\ Often overlooked in developing treatment strategies are important factors that affect outcomes in \\ asthma, namely behavioral issues such as the low rate of patient adherence to the prescribed \\ treatment regimen, inadequate physician-patient communication, and inconsistent implementa- \\ tion of evidence-based treatment guidelines. The low adherence rates among asthma patients \\ may be improved by education programs that emphasize both the potentially serious risks of this \\ disease and a team approach to treatment that includes patients and their families in developing a \\ treatment plan. Interactive physician education programs have been shown to improve guideline \\ implementation and physician communication skills. Indeed, effective physician-patient commu- \\ nication may be the key to improving guideline implementation and patient adherence to \\ treatment, resulting in meaningful decreases in asthma-related morbidity and mortality. Pediatr \\ Pulmonol. 2001; Supplement 21:26-30. ๑ 2001 Wiley-Liss, Inc.
}

Key words: asthma; guidelines; adherence; treatment; education; behavior; children.

\section{INTRODUCTION}

Why, despite advances in pharmacologic management, do treatment outcomes for asthma remain disappointing $?^{1}$ One answer may be that certain critical factors in improving asthma outcomes have been minimized in importance when developing a treatment plan. These factors involve behavioral issues including the low rate of patient adherence to a regimen, the underuse of evidencebased guidelines, and inadequate communication skills of physicians. This report will examine some of the impediments to changing these behaviors and consider strategies for overcoming these obstacles.

\section{OUTCOMES IN ASTHMA MANAGEMENT}

The National Institutes of Health (NIH) established five general goals for asthma management: no missed school or work days; no sleep disruptions; maintenance of normal activity levels; minimal or no need for emergency department visits or hospitalizations; and maintenance of normal to near normal lung function. ${ }^{2}$ Unfortunately, attaining these goals still remains elusive for many patients with asthma. The percentages of patients who meet these goals are unacceptably low. Recent surveys ${ }^{1}$ indicate that $49 \%$ of children and $25 \%$ of adults with asthma missed school or work because of asthmarelated problems. Additionally, 30\% of patients with asthma reported at least weekly nocturnal awakenings because of asthma-related breathing problems. Normal daily activities are also frequently affected by asthma. In fact, $48 \%$ of patients indicated that asthma limits their ability to participate in sports or recreational activities, $36 \%$ indicated that the disease limits their normal physical activities, and 25\% indicated that asthma interferes with normal social activities. The surveys also showed that, over a 12-month period, emergency department visits for the treatment of asthma attacks were required for $32 \%$ of the children and $19 \%$ of the adults with asthma, whereas unscheduled emergency visits to a physician's office or clinic were required by $55 \%$ of children and $36 \%$ of adults. Even more disturbing is that only $35 \%$ of patients with asthma reported having lung function testing during that same period. The survey results suggest that for too many patients, asthma is neither monitored nor controlled satisfactorily. Poor adherence to the treatment plan may at least partially explain these disappointing asthma survey results.

\section{PATIENT ADHERENCE}

Low rates of adherence, or failure to follow a prescribed treatment plan, are a major impediment to effective asthma management. ${ }^{3}$ The rate of primary nonadherence, defined as failure to even fill the medication prescription, is reported to be as high as $30 \%$ among asthma patients. ${ }^{4}$ Although adherence can be viewed from several behavioral constructs, the most widely accepted is the Health Belief Model. $^{5}$ To promote

Division of Pediatric Pulmonary Medicine, University of Michigan Medical Center, Ann Arbor, Michigan.

*Correspondence to: Randall Brown, M.D., M.P.H., Division of Pediatric Pulmonary Medicine, University of Michigan, 606 Brooks St., Ann Arbor, MI 48103. 


\section{Family members with asthma}

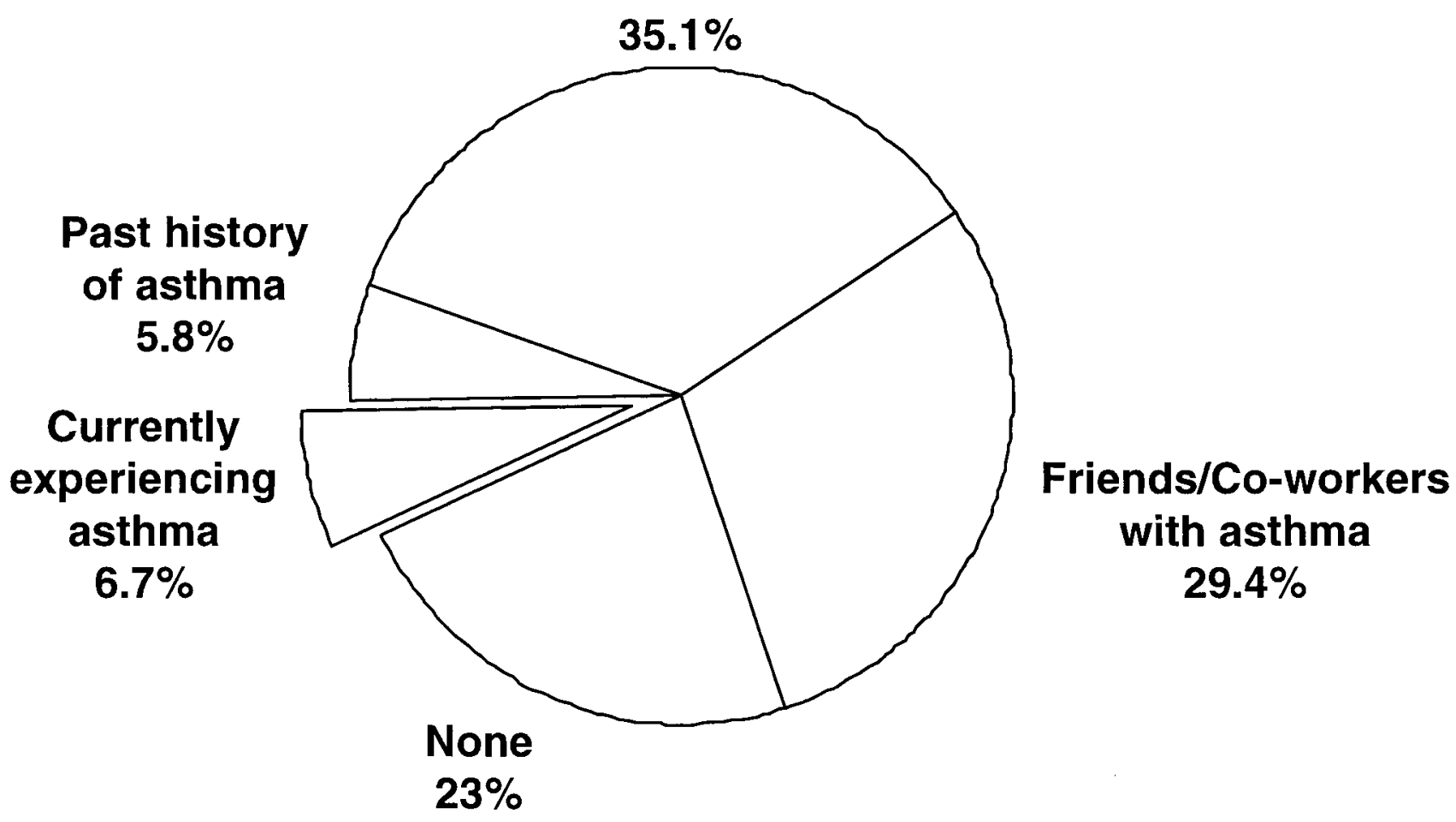

Base: All respondents (unweighted $\mathrm{N}=1000$ ). Asthma in America ${ }^{\mathrm{TM}}$. Research Triangle Park, NC: Glaxo Wellcome Inc.; 1998.

Fig. 1. Impact of asthma: $77 \%$ of the US population are directly or indirectly affected by asthma, according to this survey. Patients, family members, and friends have a vested interest in asthma management. Base: all respondents (unweighted $N=1,000$ ). Asthma in America ${ }^{\circledR}$.

adherence, this model underscores the importance of patient education. Patients and their families must understand the nature of asthma as well as the serious and sometimes fatal risks this disease poses in the absence of adequate treatment. Alarmingly, few asthma patients are aware of the true nature of their disease: only $9 \%$ of patients with asthma realize that it is fundamentally a chronic inflammatory disease. ${ }^{1}$

The patient's perceptions of the disease and the expected treatment outcomes have a major influence on the extent of adherence. If the patient underestimates the severity of asthma, or expects the medication to be difficult to administer, or that it will produce unacceptable adverse effects, adherence can be poor.

\footnotetext{
ABBREVIATIONS

CME Continuing medical education

ICS Inhaled corticosteroids

NHI National Institutes of Health
}

As a critical first step in improving adherence, the Health Belief Model emphasizes the importance of changing patients' perceptions so that they fully understand the serious nature of asthma and its potentially serious consequences. The next step involves developing a written treatment plan that clarifies the need for chronic treatment and outlines the therapeutic benefits that can be derived from adhering to the treatment plan. Finally, the barriers to adherence must be recognized and addressed. For each patient, the clinician should determine whether there are any physical, psychological, or financial obstacles that interfere with implementation of the treatment plan. Some common impediments to adherence include a stressful family environment, forgetfulness, age, peer group pressure (especially in children), and difficulty using peak flow monitoring devices.

The impact of asthma extends beyond the patient. As Figure 1 illustrates, asthma either directly or indirectly affects almost $80 \%$ of the population in the United States. Thus, it is not only the patient who has a vested interest in 
effective asthma management, but family members and friends as well. Accordingly, a team approach that includes active participation by patients and family members in defining the principal goals of treatment and developing a treatment plan can be one of the most effective means for promoting long-term adherence. The potential benefits of patient adherence, however, are maximized only if the treatment plan is consistent with evidencebased guidelines for asthma management.

\section{PHYSICIAN GUIDELINE IMPLEMENTATION}

Most primary care physicians are aware of the NIH asthma treatment guidelines, and most (80\%) believe they always, or most of the time, follow the guidelines (Fig. 2). ${ }^{1}$ In actual practice, however, guideline-recommended treatments, such as the use of inhaled corticosteroids (ICS) in patients with moderate-to-severe persistent asthma, are often underutilized (Fig. 3). ${ }^{1}$ For example, in the group of patients with severe persistent asthma, only $20 \%$ of the patients used ICS all the time. Moreover, compared with allergists and pulmonologists, pediatricians and primary care physicians were much less likely to prescribe controller medications to manage persistent asthma, as recommended by the guidelines. ${ }^{6}$

Among primary care physicians, there is an apparent disparity between advocating and actually using asthma guidelines. One reason for this disparity may be that many primary care physicians involved in asthma management are not aware of the NIH guideline content. In a study that evaluated the extent of physician knowledge about guideline content, a wide variability was seen

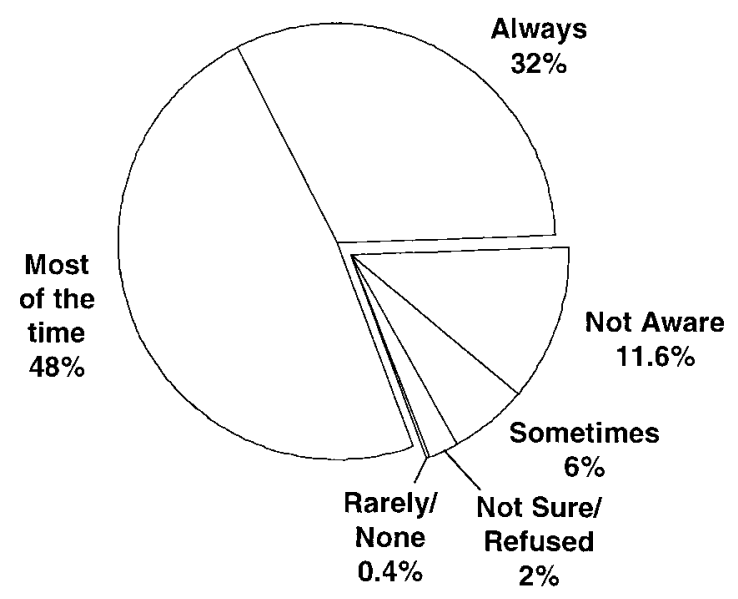

Base: All doctors $(\mathrm{N}=512)$.

Asthma in America ${ }^{\mathrm{TM}}$. Research Triangle Park, NC: Glaxo Wellcome Inc.; 1998.

Fig. 2. How often do physicians follow NIH guidelines? Most primary care physicians are aware of, and believe they follow, asthma treatment guidelines from the NIH. Compare this chart with Figure 3. Base: all doctors $(N=512)$. Asthma in America ${ }^{\circledR}$. among specialties, with family practice and internal medicine residents scoring lower than the average for all specialties evaluated. ${ }^{7}$ These results imply that primary care physicians need to become more familiar with established asthma guidelines. Although this process seems simple, it can be daunting because published guidelines are typically complex and lengthy. Condensing the guidelines into short, reader-friendly versions or distilling the most important messages and delivering those messages repeatedly can improve guideline comprehension and, ultimately, implementation.

Increasing knowledge of the guidelines is only the first step. Primary care physicians must believe that guideline implementation will not only improve outcomes but also be cost-effective. ${ }^{8}$ Important external barriers can also hinder guideline implementation. In the managed care environment, constraints placed on the amount of time a clinician can devote to each patient can hinder consideration of the treatment options available, reducing the chance that guideline-backed alternatives will be suggested and used.

\section{PHYSICIAN COMMUNICATION SKILLS}

There is a wide gulf in perception between physicians and their patients in terms of what type of asthma information has actually been communicated. According to one survey, although $70 \%$ of primary care physicians believed that they developed and presented a written action plan for asthma management to their patients, only $27 \%$ of the patients indicated they received a written action plan (see Fig. 4). ${ }^{1}$ Other similar large disparities existed between physicians' and patients' perceptions of whether peak flow meters were prescribed, lung function tests were ordered, or follow-up visits were scheduled. Obviously, primary care physicians are not effectively communicating important messages about the need for monitoring and ongoing treatment to their patients with asthma, thus undermining the very treatment goals they aim to accomplish.

\section{IMPROVING EDUCATION AND COMMUNICATION}

It could be suggested that uncontrolled asthma is a consequence more of inadequate communication than of inflammation. Although physicians cannot predict adherence on the basis of patient's age, income, education, race, gender, or profession, adherence can be predicted based on how well physicians communicate with and educate their patients. The troubling factor seems to be that most physicians believe they communicate effectively, and most patients feel that their clinician's communication and education efforts are inadequate. ${ }^{9}$

In one study, evaluating the long-term effectiveness of interactive physician education on physician guideline use and patient health, ${ }^{9} 74$ experienced pediatricians 


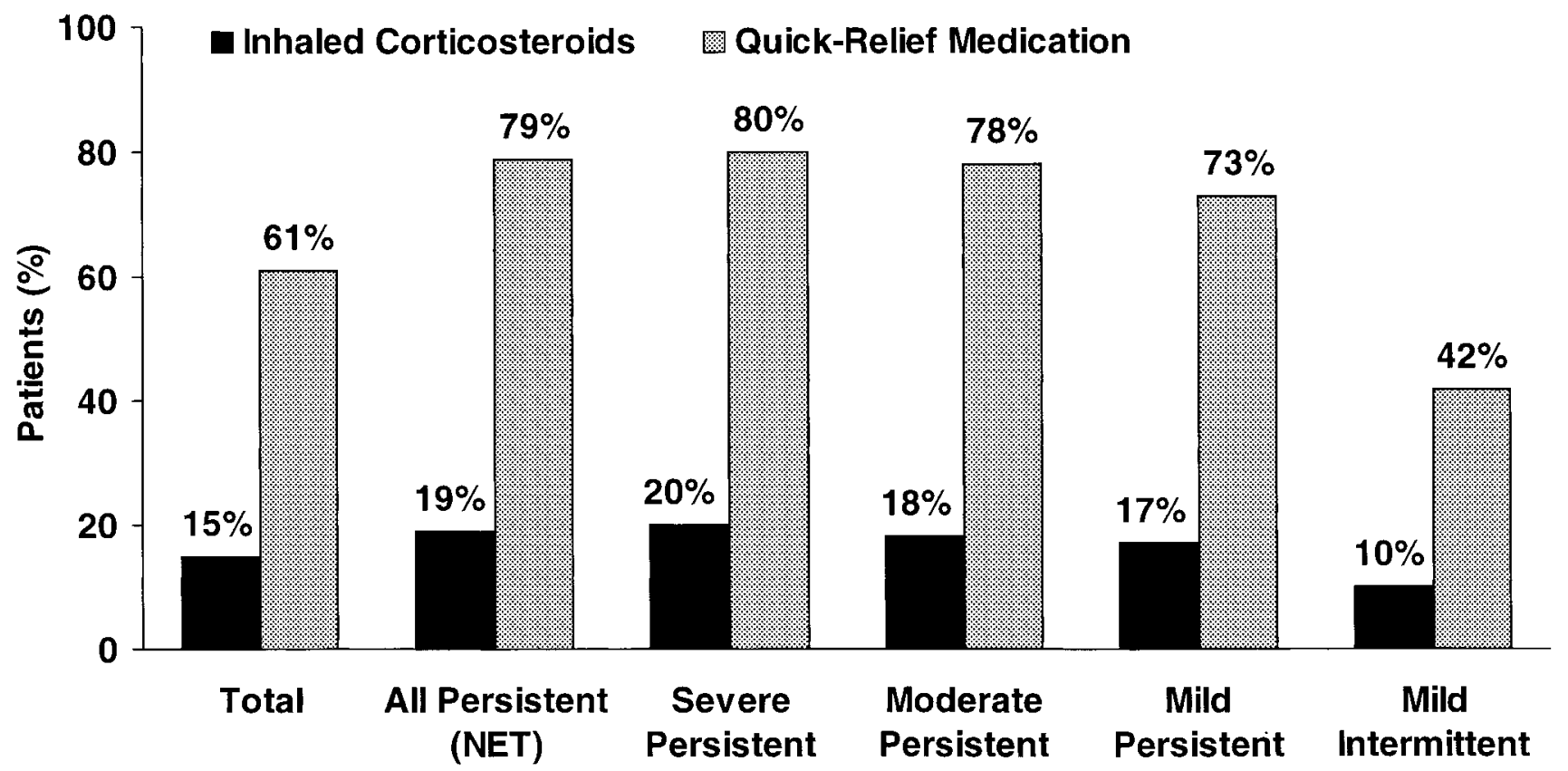

Base: All patients (unweighted $\mathrm{N}=2509$ ).

Asthma in America ${ }^{T M}$. Research Triangle Park, NC: Glaxo Wellcome Inc.; 1998.

Fig. 3. Medicines used to treat asthma by NIH severity. Treatments recommended by the NIH include use of ICS for severe persistent asthma; ICS were used by only $20 \%$ of these patients. Compare with Figure 2. Base: all patients (unweighted $N=2,509)$. Asthma in America ${ }^{\mathbb{R}}$.

participated in two 2.5-hr seminars. One of the seminars covered the use of NIH guidelines in the treatment of asthma in the context of specific case studies. ${ }^{2}$ The second seminar focused on developing effective communication and teaching skills in the clinical setting. Compared with a control group of physicians who did not participate in these training sessions, the trained physicians used asthma guidelines and provided their patients with written treatment plans. Patients of physicians who participated in the interactive seminars observed significantly fewer emergency department visits and hospitalizations due to asthma exacerbations, and the patients' families indicated that these physicians were more attentive and provided more effective asthma education. There was no significant difference, however, in the quantity of antiinflammatory medications used by patients, whether or not the physician participated in the seminars. This suggests that better communication between trained physician and patient results in more effective use of treatments (especially inhalers) and therefore a better control of asthma which is not due to use of medications alone. According to these data, when physicians participate in interactive training programs, they are more likely to implement guidelines, communicate with and educate their patients effectively, and promote patient adherence, leading to better control of the disease.

\section{CONCLUSIONS}

In asthma management, educational issues will likely move to the forefront as a means of improving physician communication, patient adherence, and treatment outcomes. Traditional continuing medical education (CME) courses obviously have not resulted in the behavioral changes needed to improve outcomes in asthma. It is questionable as to whether traditional CME courses will be sufficient to achieve these ends. The key to successful asthma management is changing the behavior of both physicians and patients.

Physician education programs that emphasize handson, interactive training can result in the behavioral changes necessary to increase the implementation of guidelines and to more effectively educate the patient about the seriousness of asthma and the importance of adherence. In addition, asthma coalition groups can use the print and electronic media to provide asthma information tailored to the needs of specific groups, expanding and reinforcing the physicians' patient education efforts.

In the current managed care environment, changing the behaviors of physician and patient can be challenging. Managed care organizations may promote treatment protocols that reduce cost but do not necessarily promote adherence or standards of care. Nevertheless, in the 


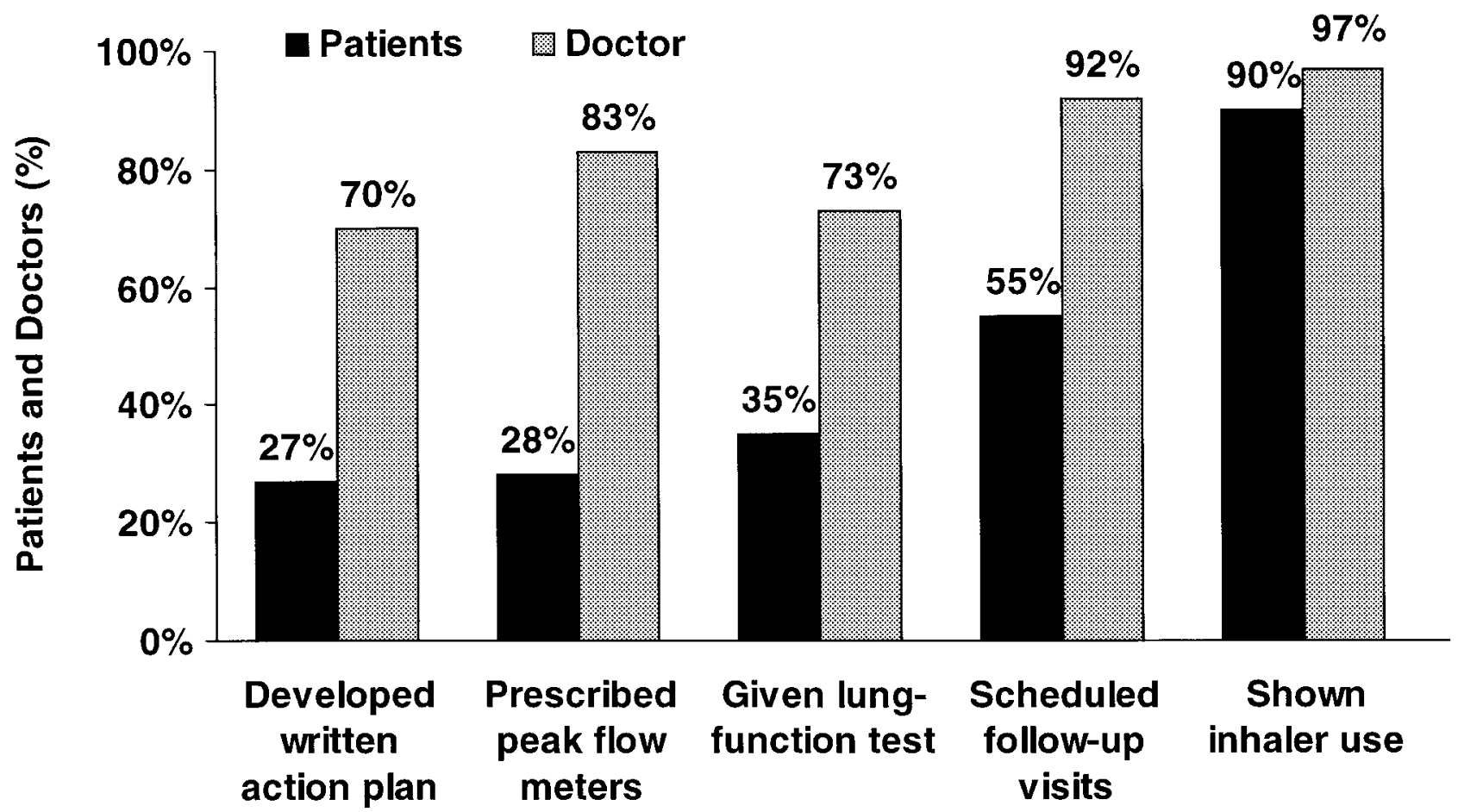

Base: All patients (unweighted $\mathrm{N}=2509$ ); all doctors ( $\mathrm{N}=512$ ).

Asthma in AmericaTM. Research Triangle Park, NC: Glaxo Wellcome Inc.; 1998.

Fig. 4. Two perspectives: patients and physicians. Although $70 \%$ of primary care physicians in a survey believed they had developed and presented a written plan for asthma management to their patients, only $27 \%$ of patients indicated they had received a written action plan. The survey also indicated other large communication "disconnects" between patients and physicians. Base: all patients (unweighted $N=2,509$ ); all doctors $(N=512)$. Asthma in America ${ }^{\mathbb{R}}$.

context of the appalling epidemiologic trends for asthma, particularly in American cities, behavioral changes among physicians and patients could well translate into meaningful decreases in asthma-related morbidity and mortality, and thus provide an opportunity for improving the cost-effectiveness of treatment as well.

\section{REFERENCES}

1. Asthma in America. Research Triangle Park, NC: Glaxo Wellcome, Inc.; 1998. http://www.asthmainamerica.com

2. National Asthma Education and Prevention Program. Expert panel report 2: clinical practice guidelines. Guidelines for the diagnosis and management of asthma. Rockville, MD: US Department of Health and Human Services, Public Health Service, National Institutes of Health, National Heart, Lung, and Blood Institute; July 1997. NIH publication no. 98-4051;1997.
3. Cochrane GM, Horne R, Chanez P. Compliance in asthma. Respir Med 1999;93:763-769.

4. Watts RW, McLennan G, Bassham I, el-Saadi O. Do patients with asthma fill their prescriptions? A primary compliance study. Aust Fam Physician 1997;26:112.

5. Janz NK, Becker MH. The Health Belief Model: a decade later. Health Educ Q 1984;11:1-47.

6. National Prescription Audit. Total asthma prescriptions. 1998.

7. Doerschug KC, Peterson MW, Dayton CS, Kline JN. Asthma guidelines: an assessment of physician understanding and practice. Am J Respir Crit Care Med 1999;159:1735-1741.

8. Cabana MD, Rand CS, Powe NR, Wu AW, Wilson MH, Abboud PA, Rubin HR. Why don't physicians follow clinical practice guidelines? A framework for improvement. JAMA 1999;282: $1458-1465$.

9. Clark NM, Gong M, Schork MA, Evans D, Roloff D, Hurwitz M, Maiman L, Mellins RB. Impact of education for physicians on patient outcomes. Pediatrics 1998;101:831-836. 